\title{
Desarrollo y evaluación de alimentos: galleta, salpor y bebida tipo horchata elaborados a partir de harinas de semillas de melón (Cucumis melo)
}

\author{
Walter Alexander Cosme-Linares ${ }^{1}$ / Blanca Estela Saravia-Arias²/ \\ Delmi Milagro Gracias-Portillo ${ }^{3}$
}

Facultad Multidisciplinaria de Ilobasco

Universidad Católica de El Salvador, El Salvador

DOI:

Recepción: 04/05/2021 Aceptación: 01/07/2021

\section{Resumen}

El trabajo consistió en el desarrollo y evaluación de alimentos: galleta, salpor ${ }^{4}$ y bebida tipo horchata, elaborados a partir de harina de semillas de melón, como una opción de diversificación en productos de consumo popular en El Salvador; al mismo tiempo, esto representa una alternativa para aprovechar la semilla del melón que, por lo general, es desechada.

Para la obtención de harina se inició con la selección de melones, extracción de semillas, secado, molido, tamizado. Posteriormente, se procedió a utilizar en diferentes formulaciones a fin de estandarizar y analizar la estabilidad en combinación con otros ingredientes. Luego se realizaron pruebas sensoriales a los productos para determinar los porcentajes de harina de melón en las diferentes recetas: 8.33\% para galleta; $5.04 \%$ para salpor y $2.02 \%$ para horchata.

Con base en el análisis físico químico realizado, la harina de semilla de melón contiene propiedades nutricionales tales como proteínas, carbohidratos, grasas de origen vegetal (ácido linoleico), las cuales fueron transmitidas a los productos elaborados, aumentando con ello el valor nutricional.

Palabras clave: Melón, semilla, salpor, galleta, harina.

\begin{abstract}
The work consisted on the development and evaluation of foods: biscuit, salpor and horchata-type drink, made from melon seed flour, as a diversification option in popular consumer products in El Salvador; at the same time, this represents an alternative to take advantage of the melon seed that, in general, is discarded.

To obtain flour, it began with the selection of melons, extraction of seeds, drying, grinding, sifting. Subsequently, it was used in different formulations in order to standardize and analyze the stability in combination with other ingredients. Sensory tests were then carried out on the products to determine the percentages of melon flour in the different recipes: $8.33 \%$ for biscuits; $5.04 \%$ for salpor and $2.02 \%$ for horchata.

Based on the physical chemical analysis carried out, melon seed flour contains nutritional properties such as proteins, carbohydrates, fats of vegetable origin (linoleic acid), which were transmitted to the processed products, thereby increasing the nutritional value.
\end{abstract}

Key words: Melon, seed, salpor, biscuit, flour.

1. Maestro en Asesoría Educativa, Investigador-Docente; email: walter.cosme@catolica.edu.sv. ORCID: https://orcid.org/ 00000003-1758-5722

2. Ingeniera Industrial, Docente-Investigador; email: blanca.saravia@catolica.edu.sv. ORCID: https://orcid.org/ 0000-0001-6647-8152

3. Ingeniera en Tecnología y Procesamiento de Alimentos, Docente-Investigador; email: delmi.gracias@catolica.edu.sv. ORCID: https://orcid.org/ 0000-0002-9844-1765

4. Pan tradicional de El Salvador, elaborado a base de harina y semejante a una galleta. Su forma puede variar dependiendo de la región en donde se elabore. 


\section{Introducción}

Se conoce que la producción de alimentos presenta grandes retos cuando se trata de ver hacia la seguridad y soberanía alimentaria. La industria alimentaria constantemente está en desarrollo; la diversidad de fórmulas en alimentos procesados como en los mínimamente procesados, el empleo de técnicas novedosas y el avance en las investigaciones para producir alimentos nutritivos con la menor cantidad de aditivos químicos, o bien, tratados mínimamente con agroquímicos son solo algunos de estos ejemplos. Acorde a la Organización de las Naciones Unidas para la Alimentación y la Agricultura (FAO, 2018), en su publicación sobre el futuro de los alimentos, todo parece reflejar que, para el año 2050 ninguna forma de producción actual podría dar abasto a las exigencias de alimentación y acceso a la misma, por parte de la población mundial. Sin embargo, acudir a nuevas alternativas de materia para el desarrollo de alimentos nuevos o poco conocidos, permite de forma positiva utilizar otras fuentes de nutrición subutilizadas como semillas y cáscaras de frutas.

El melón (Cucumis melo L.) es una planta herbácea monoica cuyo origen se presume en Asia, India y África (Giaconi, 1989); y que ahora es un cultivo de amplia difusión comercial en El Salvador. Entre los melones que tienen una mayor comercialización a ni- vel mundial se encuentran los tipos Cantaloupe (Calameño) que son reticulados, con una cubierta o cáscara en forma de red; y el Honeydew (Tuna) con cáscara lisa. Es importante reconocer otras variedades como Galia, Charentais y Piel de sapo.

La palabra "tipo" hace referencia a todo el grupo de melones que presentan una característica claramente identificable y diferenciada de los demás, en su piel, coloración de la pulpa y forma del fruto (ver tabla 1).

El melón es una de las frutas de mayor producción a nivel mundial. El continente asiático muestra mayor producción de esta cucurbitácea, con valores de más de 18 millones de toneladas métricas por año para el período 2016-2018 (FAO Statistics, s.f.). La importación de melón a El Salvador es de 15, 174. 721 kilogramos por año, lo que representa un valor FOB de $\$ 1,042,789.30$.

Esta producción a escala comercial es de 307 mz., y se encuentra localizada en su mayor parte cerca de la zona costera del país, principalmente en los departamentos de Ahuachapán, Sonsonate y La Paz. Esta producción es para consumo interno, lo que representa una oportunidad para diversificar productos derivados del mismo; además de una oportunidad de innovación y una oferta de productos con propiedades nutricionales, mediante el aprovechamiento óptimo de esta fruta (IV Censo Agropecuario 2007-2008). 


\section{Tabla 1}

Tipos de melón y sus principales características

\begin{tabular}{ll}
$\begin{array}{c}\text { Nombre de la } \\
\text { variedad }\end{array}$ & Características \\
\hline Amarillo & $\begin{array}{l}\text { Es de origen español; debe su nombre al color de su piel, mientras que su pulpa es } \\
\text { blanco-cremosa. La variedad más exportada es el melón redondo y liso. }\end{array}$ \\
\hline Honeydew & $\begin{array}{l}\text { Es conocido como melón Tuna. Posee una pulpa verde y una cáscara lisa de color } \\
\text { blanco-verdoso, tornándose amarillento durante la época de cosecha. }\end{array}$ \\
\hline Piel de sapo & $\begin{array}{l}\text { Es la variedad más conocida de entre los melones verdes; pero su peso es más } \\
\text { elevado (1.5 a } 3 \text { Kg). }\end{array}$ \\
\hline Charentais & $\begin{array}{l}\text { De origen francés, que presenta dos variedades: piel lisa y piel reticulada. En el } \\
\text { primero, el color de la piel es verde claro o ligeramente gris, dividida por suturas de } \\
\text { color verde oscuro. La variedad de piel reticulada también se presenta dividida por } \\
\text { suturas, pero estas son de color verde oscuro. }\end{array}$ \\
\hline &
\end{tabular}

\begin{tabular}{ll} 
Galia & $\begin{array}{l}\text { Posee forma redondeada y piel de color verde, que evoluciona a amarilla en la } \\
\text { madurez, con un reticulado fino. }\end{array}$ \\
\hline Cantaloupe $\quad \begin{array}{l}\text { Es de origen norteamericano y el más producido. Es esférico y presenta un grueso } \\
\text { reticulado en toda la superficie. }\end{array}$
\end{tabular}

Nota. Información retomada de Manual de manejo agronómico para el cultivo del melón, 2017.

El melón es una de las frutas de mayor pro- Esta producción a escala comercial es de 307 ducción a nivel mundial. El continente asiáti- $\quad$ mz., y se encuentra localizada en su mayor co muestra mayor producción de esta cucurbitácea, con valores de más de 18 millones de toneladas métricas por año para el período 2016-2018 (FAO Statistics, s.f.). La importación de melón a El Salvador es de 15, 174. 721 kilogramos por año, lo que representa un valor $\mathrm{FOB}^{5}$ de $\$ 1,042,789.30$. parte cerca de la zona costera del país, principalmente en los departamentos de Ahuachapán, Sonsonate y La Paz. Esta producción es para consumo interno, lo que representa una oportunidad para diversificar productos derivados del mismo; además de una oportunidad de innovación y una oferta de produc-

5. Entiéndase como el precio de venta de los bienes embarcados, puestos en el medio de transporte, sin incluir valor de seguro y flete. 
tos con propiedades nutricionales, mediante el aprovechamiento óptimo de esta fruta (IV Censo Agropecuario 2007-2008).

\section{Valor nutricional de las semillas de Cucu- mis melo}

El melón es una fruta que se comercializa en su mayoría para ser consumida en forma de bebida, y poco se conoce sobre la utilidad de la semilla como insumo dentro de alimentos procesados. En países como México, la semilla de melón se ha utilizado para preparar algunas bebidas, tales como la horchata; aunque este tipo de bebida a base de este elemento recientemente ha dejado de ser consumida.

Las características de la semilla de melón muestran un contenido proteico del 24\% al 27\%, siendo que estas proteínas pueden contener faseolinas, empleadas dentro de fórmulas cárnicas; de galletas y de pan ( $\mathrm{Ra}$ mírez-Pimentel et al, 2016). Pensar en diversas formulaciones alimentarias permitiría aprovechar un recurso poco utilizado y con importante valor nutricional.

Las semillas de melón, por ser un reservorio natural para dar origen a una nueva planta, conservan en su interior diversidad de compuestos como glúcidos, vitaminas y minerales. En efecto, algunos estudios han encontrado en el contenido de estas semillas algunos minerales como el potasio, fósforo, magnesio y azufre, siendo semejante a las semillas de frijol y maíz. La proteína presente en la semilla de melón es soluble de $\mathrm{pH}$ ácido, neutro o alcalino, siendo favorable para su uso en formulaciones alimentarias (Ramírez-Pimentel et al., 2016).

El valor nutricional estudiado en las semillas de melón es el atributo destacado para el desarrollo de alimentos funcionales, sobre todo cuando el principal interés es un alimento que contribuya a mantener equilibradas las funciones metabólicas de control de peso. Tal es así que los ácidos grasos similares a los del aceite de soya y girasol lo hace aún más preciado para incluirlo en las formulaciones de alimentos diversos (Ramírez-Pimentel et al, 2016). Las propiedades antioxidantes, antiinflamatorias, antidiabéticas y anti ulcerosos se atribuyen a la presencia de incompuestos en la cáscara, semillas y pulpa de la fruta (Silva et al., 2020).

\section{Materiales y Métodos}

La investigación se llevó a cabo en el laboratorio de investigación y en la planta piloto perteneciente a la Escuela de Alimentos del Técnico en Lácteos y Cárnicos de la Facultad Multidisciplinaria de Ilobasco de la Universidad Católica de El Salvador, ubicada en el departamento de Cabañas, El Salvador.

Para este estudio, se buscó elaborar harina a base de semilla de melón, con el objetivo de aprovechar un posible subproducto, el cual 
no está siendo utilizado y podría aportar algo a una receta ya existente.

A continuación, se muestra el listado de equipos utilizados para la obtención de harina de semilla de melón:

- Horno de convección computarizado (HCU) Cuchillos

- Tablas para picar

- Contenedores plásticos

- Molino nixtamalizado

- Balanza

- Tamizadoras

- Bandejas americanas de aluminio $(18 " x 26 ")$

\section{Proceso de obtención de harina}

\section{a. Método de extracción de semilla}

Para realizar el corte de la fruta se utilizaron cuchillos de acero inoxidable. La semilla se extrajo específicamente de forma manual, con esto se previó cualquier tipo de reacción química por efecto de la acidez de las semillas al entrar en contacto con el material del cuchillo. Se probaron dos métodos de extracción: el primero consistió en retirar la membrana de forma manual; luego, lavar las semillas con abundante agua purificada para eliminar la sustancia gelatinosa que las recu- bre. En el segundo método también se extrajo de forma manual; posteriormente, se colocó en un recipiente con agua y se dejó en reposo a temperatura ambiente durante 24 horas. Esto con el objetivo de permitir un inicio de fermentación y que, la membrana junto con el gel que recubría las semillas se separara por sí misma. Este segundo método no se recomienda para fines alimenticios, ya que la semilla adquiere sabor amargo.

\section{b. Método de secado de semilla}

Se realizó un secado en horno, en donde se ajustó la temperatura a $65^{\circ} \mathrm{C}$ durante ocho horas de tiempo. Las semillas se esparcieron en bandejas americanas de aluminio de $18 \times 26$ pulgadas, además fueron cubiertas por otra bandeja para evitar que las semillas no se esparcieran por la ventilación del horno de convección. Una vez agotado el tiempo se procedió a realizar análisis de humedad en la termo balanza, la cual arrojó un valor de 6.5\% de humedad para las semillas (ver tablas 2-4).

\section{Resultados y Discusión}

Durante la elaboración de los productos, en las primeras producciones se obtuvo un sabor aceptable según características organolépticas; a excepción de la textura, la cual resultó con alto grado de dureza, para lo cual se trabajó en la formula hasta obtener un producto con una textura suave. 


\section{Tabla 2}

Formulaciones evaluadas de galleta

\begin{tabular}{ccc}
\hline Ingredientes & Porcentaje utilizado en fórmula & Porcentaje utilizado en fórmula \\
\hline $\begin{array}{c}\text { Harina de semilla de } \\
\text { melón }\end{array}$ & $\mathbf{1}$ & $\mathbf{2}$ \\
\hline Harina de trigo & $44.85 \%$ & $8.33 \%$ \\
\hline Margarina & --- & $46.66 \%$ \\
\hline Azúcar & $23.88 \%$ & $20.83 \%$ \\
\hline Huevo & $14.9 \%$ & $16.16 \%$ \\
\hline Leche en polvo & $8.9 \%$ & $6.66 \%$ \\
\hline Yema de huevo & $4.48 \%$ & --- \\
\hline Sal & $2.2 \%$ & --- \\
\hline Polvo de hornear & $0.33 \%$ & --- \\
\hline Vainilla & $0.31 \%$ & --- \\
\hline Total & --- & $0.83 \%$ \\
\hline
\end{tabular}

\section{Tabla 3}

Formulaciones evaluadas de salpor

\begin{tabular}{ccc}
\hline Ingredientes & Porcentaje utilizado en fórmula & Porcentaje utilizado en fórmula \\
\hline $\begin{array}{c}\text { Harina de semilla de } \\
\text { melón }\end{array}$ & $\mathbf{1}$ & $\mathbf{2}$ \\
\hline Harina suave & $53 \%$ & $5.4 \%$ \\
\hline Harina de arroz & --- & $30.64 \%$ \\
\hline Azúcar & --- & $18.38 \%$ \\
\hline Huevo & $26.8 \%$ & $18.38 \%$ \\
\hline Margarina & $11.8 \%$ & $4.32 \%$ \\
\hline Leche en polvo & $6.5 \%$ & $18.38 \%$ \\
\hline Polvo para hornear & --- & $3.06 \%$ \\
\hline Vainilla & $0.7 \%$ & $0.75 \%$ \\
\hline Sal & --- & $0.54 \%$ \\
\hline Canela & $1.0 \%$ & $0.1 \%$ \\
\hline Total & $0.1 \%$ & ---- \\
\hline
\end{tabular}


Tabla 4

Formulaciones evaluadas de horchata

\begin{tabular}{|c|c|c|c|c|c|c|}
\hline Ingredientes & $\begin{array}{l}\text { Porcentaje } \\
\text { utilizado en } \\
\text { fórmula } 1\end{array}$ & $\begin{array}{c}\text { Porcentaje } \\
\text { utilizado en } \\
\text { fórmula } 2\end{array}$ & $\begin{array}{c}\text { Porcentaje } \\
\text { utilizado en } \\
\text { fórmula } 3\end{array}$ & $\begin{array}{c}\text { Porcentaje } \\
\text { utilizado en } \\
\text { fórmula } 4\end{array}$ & $\begin{array}{c}\text { Porcentaje } \\
\text { utilizado en } \\
\text { fórmula } 5\end{array}$ & $\begin{array}{c}\text { Porcentaje } \\
\text { utilizado en } \\
\text { fórmula } 6\end{array}$ \\
\hline Agua & $55.74 \%$ & $62.46 \%$ & $80.64 \%$ & $81 \%$ & $81 \%$ & $76.33 \%$ \\
\hline Leche condensada & $17.5 \%$ & --- & --- & --- & --- & --- \\
\hline Hielo & $13.37 \%$ & $14.99 \%$ & --- & -- & --- & -- \\
\hline $\begin{array}{l}\text { Horchata en polvo } \\
\text { (Marca Lorena) }\end{array}$ & --- & -- & --- & $9.2 \%$ & -- & $9.16 \%$ \\
\hline $\begin{array}{c}\text { Mezcla de semilla } \\
\text { para horchata }\end{array}$ & -- & -- & --- & -- & $9.2 \%$ & --- \\
\hline Azúcar & $8.36 \%$ & $11.8 \%$ & $12.09 \%$ & $7.7 \%$ & $7.7 \%$ & --- \\
\hline Almendras & --- & $6.4 \%$ & --- & --- & --- & $14.5 \%$ \\
\hline $\begin{array}{l}\text { Harina de semilla } \\
\text { de melón }\end{array}$ & $4.37 \%$ & $4.3 \%$ & $7.25 \%$ & $2.02 \%$ & $2.02 \%$ & --- \\
\hline Limón & --- & $0.03 \%$ & -- & --- & --- & --- \\
\hline Vainilla & $0.27 \%$ & --- & --- & --- & --- & --- \\
\hline Canela en raja & $0.16 \%$ & --- & --- & --- & --- & --- \\
\hline Canela molida & --- & $0.006 \%$ & --- & --- & --- & --- \\
\hline Total & $99.77 \%$ & $99.986 \%$ & $99.98 \%$ & $99.92 \%$ & $99.92 \%$ & $99.99 \%$ \\
\hline
\end{tabular}

Una vez elaborados los productos se sometieron a una serie de análisis físicos, sensoriales y microbiológicos para evaluar la calidad y condiciones de higiene en los cuales se procesaron. Para los análisis microbiológicos se utilizó el método de placas deshidratada o placa Petrifilm de recuentos de mohos y levaduras, Salmonella spp, Escherichia Coli. Se tomaron muestras de las producciones para posteriormente realizar los análisis.

Al realizar el análisis sensorial durante la prueba organoléptica realizada a la receta tradicional de horchata, con diferentes porcen- tajes de harina de semilla de melón, los datos arrojaron un olor de $32.2 \%$; un color del $33.3 \%$ y un sabor del $34 \%$.

\section{Resultado de análisis microbiológicos}

En las tablas 5 y 6 se muestran los resultados del análisis microbiológico y análisis físico químico. Es evidente la ausencia de microorganismos patógenos y un alto porcentaje de proteína, lo que significa que es un producto apto para el consumo humano, que aporta nutrientes esenciales para una dieta saludable. 


\section{Tabla 5}

Análisis microbiológico de harina de semilla de melón

\begin{tabular}{|c|c|c|c|c|c|}
\hline Determinación & PA & $\begin{array}{l}\text { UFCl } \\
\text { g }\end{array}$ & $\begin{array}{l}\text { MNP } \\
/ g\end{array}$ & Método & Referencia \\
\hline $\begin{array}{l}\text { M006 Escherichia } \\
\text { Coli }\end{array}$ & & & $<3$ & $\begin{array}{l}\text { Tubos de fermentación } \\
\text { múltiple }\end{array}$ & $\begin{array}{c}\text { US FDA, BAN } \\
\text { online, } \mathrm{Ch}\end{array}$ \\
\hline $\begin{array}{c}\text { M010 Recuento de } \\
\text { Mohos y } \\
\text { Levaduras }\end{array}$ & & $<10$ & & Vertido en placa & $\begin{array}{c}\text { US FDA, BAN } \\
\text { online, } \mathrm{Ch}\end{array}$ \\
\hline $\begin{array}{l}\text { M011 Salmonella } \\
\text { sp }\end{array}$ & Ausencia & & & Presencia/ ausencia & $\begin{array}{c}\text { US FDA, BAN } \\
\text { online, } \mathrm{Ch}\end{array}$ \\
\hline
\end{tabular}

Nota. Datos obtenidos del Laboratorio de análisis microbiológico, Fundación Salvadoreña para el Desarrollo Económico y Social (FUSADES, s.f.).

\section{Tabla 6}

Análisis físico químico de harina de semillas de melón

\begin{tabular}{cccc}
\hline Determinación & $\begin{array}{c}\text { Resultados en diversas } \\
\text { unidades }\end{array}$ & Método & Referencia \\
\hline C003 Proteína & $18.24 \mathrm{~g} / 100 \mathrm{~g}$ muestra & Kjeldahl Modificado & AOAC, 16 Ed.1995 \\
\hline $\begin{array}{c}\text { C004 Grasa muestra } \\
\text { húmeda }\end{array}$ & $28.25 \mathrm{~g} / 100 \mathrm{~g}$ muestra & Babcock Modificado & $\begin{array}{c}\text { 8td M Dalry Prods } \\
17 \text { th Ed }\end{array}$ \\
\hline C006 Ceniza & $3.49 \%$ & Gravimétrico & AOAC, 16 Ed.1995 \\
\hline C019 Carbohidratos & $49.07 \%$ & Calculo por diferencia & $\begin{array}{c}\text { M enchu, MT } \\
\text { INCAP 2006 }\end{array}$ \\
\hline C046 Humedad & $0.95 \%$ & $\begin{array}{c}\text { Analizador } \\
\text { HalogenonHR73 }\end{array}$ & $\begin{array}{c}\text { Mettler } \\
\text { Toledo,02/2001 }\end{array}$ \\
\hline C047 Sodio & $69.11 \mathrm{mg} / 100 \mathrm{~g}$ & $\begin{array}{c}\text { Espectrofotometría } \\
\text { absorción atómica }\end{array}$ & AOAC, 16Ed.1995 \\
\hline
\end{tabular}

Nota. Datos obtenidos del Laboratorio de análisis microbiológico, Fundación Salvadoreña para el Desarrollo Económico y Social (FUSADES, s.f.).

La horchata de semilla de melón aporta 167 kilocalorías, lo que representa un producto saludable de acuerdo a la Norma Salvadoreña Obligatoria (Comités Técnicos de Normalización del Consejo Nacional de Ciencia y Tecnología, 2021). Las semillas de melón tienen un efecto positivo en la salud, así lo confirma el sitio de divulgación científica del Tecnoló- gico de Monterrey (Garza, 2021). Según se observa en la tabla, su aporte energético se debe a la cantidad de ácido linoleico; un ácido graso esencial poliinsaturado que se puede encontrar en los aceites de origen vegetal. Este es de gran beneficio para el organismo, además de poseer otras características que se representan como proteína y carbohidratos. 
Tabla 7

Información nutricional de horchata de semilla de melón

\begin{tabular}{ccc}
\hline & Cantidad por porción & \%VRN \\
\hline Energía $(\mathrm{Kj})$ & 700 & 8 \\
\hline Grasa total $(\mathrm{g})$ & 9 & 14 \\
\hline Grasa saturada $(\mathrm{g})$ & 2 & 8 \\
\hline Carbohidratos $(\mathrm{g})$ & 16 & 5 \\
\hline Sodio $(\mathrm{mg})$ & 20 & 1 \\
\hline Proteína total $(\mathrm{g})$ & 6 & 12 \\
\hline
\end{tabular}

Nota. Información calculada para una porción de 32g (3 cucharadas); estableciendo una porción por envase de $14 x$. Los porcentajes que se muestran están basados en valores diarios de una dieta de 2000 calorías. ${ }^{\star}$ El porcentaje de valor diario ha sido calculado en base a referencia del RTCA. Datos obtenidos del Laboratorio de análisis microbiológico, Fundación Salvadoreña para el Desarrollo Económico y Social (FUSADES, s.f.).

\section{Conclusiones}

Afrontar los cambios inciertos del futuro climático, ambiental y culinario es algo que debe preocuparnos a todos $y$, por ende, trabajar para tener soluciones eficientes. El estudio se llevó a cabo poniendo a prueba las recetas populares, simples de elaborar, y carentes de información nutricional del recetario gastronómico de El Salvador; agregándoles la harina de semillas de melón elaborada por el equipo de investigación dentro del laboratorio de la Facultad Multidisciplinaria de Ilobasco en el departamento de Cabañas.

Los resultados de los análisis nutricionales elaborados por el laboratorio de la unidad de análisis físico químicos de la Fundación Salvadoreña para el Desarrollo Económico y Social (FUSADES) comprobaron la exis- tencia de un $18 \%$ de proteína en la harina de melón elaborada por el equipo investigador de la Universidad Católica de El Salvador. Los resultados se encuentran en concordancia con lo expuesto por Ramírez-Pimentel et al (2016), en cuanto a sus estudios de caracterización de las proteínas de la semilla de melón.

El porcentaje de humedad presente fue de 6.5\% (según analizador de humedad y termo balanza), esto después de ocho horas de deshidratación para poder elaborar la harina. Este porcentaje de humedad es determinante para facilitar el molido de la semilla. Al momento de moler es importante agregar 3\% de agua para facilitar la trituración y el paso por los discos del molino. Dependiendo del resultado, puede ser necesario colocar la masa en horno deshidratador a $65^{\circ} \mathrm{C}$ por cuatro horas. 
Acorde al artículo de divulgación científica del Tecnológico de Monterrey (Garza, 2021), las semillas de melón tienen propiedades nutricionales que protegen al corazón, y contienen altas dosis de ácido linoleico; el cual es una sustancia que mejora el funcionamiento neuronal. Al incluir la harina de semillas de melón como ingrediente en las formulaciones de galletas, salpor y horchata, este componente brinda un valor nutricional adicional, ya que contiene proteínas y carbohidratos. Conjuntamente, durante los procesos de producción, se pudo observar que la harina de melón se combina favorablemente con otros ingredientes, aunque se mezcló con otro tipo

\section{Referencias} NUTRICIO.pdf parencia.gob.sv cle/view/2546/2574 de grasas vegetales, este no modificó las características sensoriales de los productos.

A pesar de que en el estudio no se estableció un objetivo relacionado a la extracción de aceite de semilla de melón, el uso que este puede tener representa un tema de investigación a futuro, ya que se tiene conocimiento de algunos datos relacionados al uso del aceite de melón, de acuerdo al análisis nutricional realizado en el laboratorio de la Fundación Salvadoreña para el Desarrollo Económico y Social (FUSADES) y de Kalahari melón oil sedes, que es un referente en la industria de la cosmética y cuidado personal.

Comités Técnicos de Normalización del Consejo Nacional de Ciencia y Tecnología, C. ( 2021). Directrices del Códex Alimentarius sobre etiquetado nutricional. NSO CODEX CAC/GL 2 https://www.defensoria.gob.sv/images/stories/varios/NORMAS/ETIQUETADO/DIRECTRICES\%20DEL\%20CODEX\%20ALIMENTARIUS\%20SOBRE\%20ETIQUETADO\%20

Espinosa-Garza, G. (2021). Transferencia TEC. Tecnológico de Monterrey. https://transferencia.tec.mx/2018/08/16/comprobado-las-semillas-de-melon-protegen-el-corazonMinisterio de Agricultura y Ganadería (2020). Anuario de Estadísticas Agropecuarias. www.trans-

Juárez-Hernández, M. S.; Escobedo-Ocádiz, L.; Zarco-Ortíz, M. D. y Arias-Rico, J. y Ramírez-Moreno, E. (2017). Empanizador de carnes elaborado a partir de semillas de Cucumis melo (melón) "Kukus - Empanizador". Educación y Salud Boletín Científico de Ciencias de la Salud del ICSa, 5 (10) https://repository.uaeh.edu.mx/revistas/index.php/ICSA/arti- 
Mian-hao, H. y Yansong, A. (2007). Characteristics of some nutritional composition of melon (Cucumis melo hybrid 'ChunLi') seeds. International journal of food science \& technology, 42(12), 1397-1401.

Ministerio de Agricultura y Ganadería (2020). Censos Agropecuarios. http://www.mag.gob. sv/direccion-general-de-economia-agropecuaria/estadisticas-agropecuarias/censos-agropecuarios.

Normativa Salvadoreña Obligatoria NSO 67.18.01:01 (2001). Productos de Alimenticios. Bebidas no carbonatadas sin alcohol, especificaciones. https://www.defensoria.gob.sv/images/stories/varios/NORMAS/BEBIDAS/nso67.18.01.01NO\%20CARBONATADAS\%20SIN\%20 ALCOHOL.pdf.

Normativa Salvadoreña Obligatoria NSO 67.30.01:04 (2004). Productos de Panadería, clasificación y especificaciones del pan dulce. https://www.defensoria.gob.sv/images/stories/varios/NORMAS/PANADERIA/PRODUCTOS\%20DE\%20PANADERIA.\%20CLASIFICACION\%20Y\%20ESPECIFICACIONES\%20DEL.pdf.

Organización de las Naciones Unidas para la Alimentación y la Agricultura (2018). The future of food and agriculture - Alternative pathways to 2050. https://www.fao.org/3/I8429EN/ i8429en.pdf

Organización de las Naciones Unidas para la Alimentación y la Agricultura (2020). Estadísticas de producción y calidad de cultivos 2017. Roma, Italia. http://www.fao.org/faostat/es/\#data/QC.

Ramírez-Pimentel, J. G. et al ( 2021). Caracterización de las proteínas de reserva y contenido mineral de semilla de melón (Cucumis melo L.). Revista Mexicana de Ciencias Agrícolas, 7 (7); 1667-1678. http://www.scielo.org.mx/scielo.php?pid=S2007-09342016000701667\&script=sci_abstract.

Recetas salvadoreñas. https://www.recetassalvadorenas.com/receta/salpores-de-arroz. 